\title{
Long-term results of the Choyce anterior chamber lens implants Marks V, VII, and VIII
}

\author{
JOHN L. PEARCE \\ Redditch, Worcs
}

This paper continues the assessment of the long-term merits and problems of intraocular acrylic lenses which was started by a consideration of the value of the Binkhorst iris clip lens in aphakia by Pearce (1972). The reduced degree of aniseikonia and the better opportunity for binocular vision when uniocular aphakia is corrected by an intraocular lens implant was emphasized by Girard, Friedman, Moore, Blau, Binkhorst, and Gobin (rg62) and the rather high failure rate of contact lens use in aphakia has been reported by Bonnet, Massin, and Gerhard (1966), Martin Walker (quoted by Davies and Panter, 1968), and Mills and Lewis (197r). Ruben (1971), in considering soft contact lenses, noted that in adult aphakics tolerance of reduced optic lenses is very good, but management, especially in lens removal, often proves difficult. Kelly and Butler (1971), in their series of cases of non-traumatic bilateral cataract using the offset contact lens, found 20 per cent. of patients unable to wear the contact lens. Stanworth (1972), in his series of 21 unilateral aphakic patients, found that five had abandoned or almost abandoned wear of their perilimbal contact lens after some years' wear. Despite the advantages of successful intraocular acrylic lens implantation in aphakia, fear of long-term complication has denied the procedure universal acceptance. Barraquer (1970) stated that, because of various complications, he had removed some 200 out of 400 anterior chamber lenses.

Choyce (1964) summarized the theoretical dangers to the eye after anterior chamber lens implantation as follows:

(I) Damage to the corneal endothelium resulting in endothelial corneal dystrophy;

(2) Chronic iritis with, or without, secondary glaucoma;

(3) Progressive fibrosis of the uveoscleral meshwork resulting in glaucoma from obstruction to aqueous flow;

(4) Late dislocation of the implant into the vitreous or suprachoroidal space;

(5) Sympathetic ophthalmitis

Address for reprints: J. L. Pearce, Ch.M., D.O., Smallwood Hospital, Redditch, Worcs.
However, in 1970, he concluded that complications of Mark VIII surgery were almost entirely confined to the first postoperative year and that the Mark VIII series when compared to the Mark I should remain trouble-free for the rest of the patient's natural life. In view of the divergence of opinion as to the advisability of implantation of anterior chamber lenses in cases of aphakia, it was felt that an independent report on a series of such patients in which an anterior chamber lens had been in situ for some years would be of value. With this in mind the author approached Mr. Choyce who agreed to an independent examination of his own cases.

It is not intended to compare directly the results in this paper with the findings of Pearce (1972) in his survey of Binkhorst's results with his iris clip lens, as Choyce favours two-stage implantation, whereas Binkhorst uses a one-stage procedure.

The theoretical advantage of the Choyce Mark VIII anterior chamber angle fixation type of lens over the iris fixation pupillary lens of Binkhorst include:

(I) After the initial cataract extraction, corneal curvature, axial length, lens plane assessment, visual acuity, and refraction can be accurately assessed so that the optical part of the implant can be made accordingly. (This can also be done with the pupillary lens if two-stage surgery is contemplated.)

(2) Large colobomas of the iris can be covered by an anterior chamber lens using a coloured haptic, whereas the pupillary lens requires at least two-thirds of the iris to be present and even then an iris stitch has to be used to reconstruct the pupil: this may require considerable technical expertise.

(3) Implantation is technically simpler, especially if there is a vitreous knuckle or free vitreous in the anterior chamber.

(4) Dilatation of the pupil can be effected without fear of dislocation of the implant for examination of the periphery of the retina; this is of especial importance in the event of a retinal detachment.

(5) There is little, if any, likelihood of dislocation of the implant, whereas Pearce (1972) found that some I5 per cent. of Binkhorst's series of iris clip lenses dislocated. However, in a current review of my own series of 136 cases in which the iris clip lens had been sutured by one iris stitch, the incidence of dislocation 
was reduced to 4.8 per cent., and no case required intraocular manipulation to reposition the lens in the pupil.

Amongst the theoretical disadvantages of the anterior chamber lens compared with the pupillary type of implant are:

(i) The corneal diameter has to be accurately measured in the meridian of implantation to ensure that the implant fits snugly into the angle of the anterior chamber, whereas the standard iris clip lens fits all cases.

(ii) An anterior chamber lens is nearer the corneal endothelium and is lodged in the angle of the anterior chamber. There would therefore seem to be an increased danger of corneal endothelial dystrophy and peripheral anterior synechiae formation which, if extensive, could give rise to secondary glaucoma.

(iii) Secondary implantation of an anterior chamber lens requires, an additional incision into the anterior chamber which could increase the peripheral anterior synechiae formation and the possibility of endothelial changes. In addition, secondary implantation necessitates a second anaesthetic which increases morbidity and mortality in an older age group.

(iv) Problems with binocular vision could arise in the interim period between the primary cataract extraction and the secondary implantation of the implant.

\section{Selection of cases}

Patients who lived within reasonable travelling distance from Southend were selected in chronological order. 79 patients were contacted. Six had died and seventeen could not attend for a variety of reasons including moving away from the district and ill health. A total of 56 patients attended for examination. In all cases surgery had been performed by $\mathrm{Mr}$. Choyce.

\section{Surgical technique of Mr. Choyce}

It is not proposed to consider this in detail. His basic two-stage implantation technique currently includes:

\section{(a) Primary cataract surgery}

An intracapsular cataract extraction using a Graefe section with a conjunctival flap; forceps cataract extraction with or without zonulolysis; peripheral iridectomies and closure of the incision with postplaced 7/o chromic catgut corneo-scleral sutures. The anterior chamber is re-formed with air.

(b) Secondary implantation of the anterior chamber lens

Some 3 months later, when the eye is satisfactorily healed and aphakic refraction and visual acuity have been assessed, the corneal diameter is measured in the implantation meridian and an appropriate implant ordered. A temporal Graefe incision is made in the cornea, the implant is inserted, several peripheral iridectomies are performed (in some cases also a basal peripheral iridectomy), and the wound is closed with four postplaced chromic catgut sutures. The anterior chamber is re-formed with air. Subconjunctival Dexamethasone is injected and topical steroids and mydriatics are used in the postoperative period as necessary. All surgery is performed under general anaesthesia.

Over the years some modification in technique has occurred, including the use of other corneo-scleral sutures, such as virgin silk.

\section{Material}

Of the 56 patients examined, 23 had bilateral implantations, so that 79 eyes had Choyce anterior chamber lenses. There were 42 female and 37 male eyes, of which 39 were right and 40 left. Of these 79 eyes, eleven were fitted with the earlier Choyce Mark V and Mark VII anterior chamber implants; these have been examined as fully as the Choyce Mark VIII implants and the relevant findings on this small series is discussed at the end of this paper.

\section{MARK VIII CASES}

There were 68 eyes; the average age at examination was 69 years and the average time since implantation of the prosthesis was 5.5 years (range $\mathrm{I}$ to 8 ).

\section{METHOD OF EXAMINATION}

All cases were examined by the author. The investigation included:

(I) Visual acuity with and without correcting spectacles. Refraction where necessary.

(2) Examination of the anterior segment of the eye with the Haag-Streit 900 slit-lamp microscope, followed by applanation tonometry, and the angle appearance through $360^{\circ}$ using the Goldmann single-mirror gonioprism. The relationship of the implant to the structures of the angle and the corneal endothelium was noted.

(3) Fundoscopy with the direct and, in certain cases, the indirect ophthalmoscope.

(4) Orthoptic assessment.

(5) Examination of the patients' hospital records and Mr. Choyce's private records to note the past history of the patient, including the aphakic visual acuity before implantation, and any operative and postoperative problems.

OPERATIVE COMPLICATIONS AT IMPLANTATION OF CHOYGE MARK VIII ANTERIOR CHAMBER LENSES

Vitreous loss appeared to be the only serious operative complication of implantation of the prosthesis. This occurred in five out of 68 eyes, an incidence of $7 \cdot 3$ per cent. It was generally a small loss and occurred in the following eyes:

Gase I Small vitreous loss at implantation. Corrected visual acuity $6 / 6,7$ years 8 months after surgery. 
Case 2 Corrected visual acuity 6/9, I year 5 months after surgery.

Case 3 Corrected visual acuity 6/6, over 8 years after surgery.

Case 4 Visual acuity 6/6, 7 years io months after surgery.

Case 5 Visual acuity $6 / 9,4$ years 10 months after surgery.

The vitreous loss does not appear to have reduced the visual result markedly in the above cases. Of course, vitreous loss at surgery is more likely if the anterior hyaloid membrane is ruptured or if there is a large vitreous knuckle in the anterior chamber.

\section{POSTOPERATIVE COMPLICATIONS AFTER} IMPLANTATION

The possibility of severe, immediate postoperative uveitis is reduced by the injection of subconjunctival steroids at the time of surgery.

The following serious complications occurred in the immediate postoperative period:

Case 6 Shallow anterior chamber after surgery. Visual acuity now $6 / 6$, I year 7 months later.

Case 7 An early postoperative hyphaema occurred in this eye which had a secondary cataract after heterochromic cyclitis. Visual acuity 6/12, 7 years after surgery.

Case 8 Postoperative keratitis and uveitis. The eye now shows corneal dystrophy with vitreous fibrosis and the visual acuity is counting fingers only.

Case 9 This eye developed uveitis 6 months after the operation with iris bombé followed by secondary glaucoma, necessitating a further basal iridectomy. The eye is irritable with a membrane from the lens to the cornea. Corrected visual acuity $6 / 36$.

The two cases of uveitis have seriously affected the patients' visual result despite the use of systemic and topical steroids.

\section{SERIOUS CORNEAL GOMPLICATIONS}

Fear of serious corneal complications is the main reason why intraocular acrylic lens implants have not been generally accepted, especially in view of the relatively low incidence of bullous keratopathy in routine cataract surgery. However, it should not be forgotten that bullous keratopathy does arise even in uncomplicated cataract surgery.

\section{SERIOUS CORNEAL GHANGES}

Bullous keratopathy occurred in one eye, an incidence of just over I per cent. This patient (Case Io) had an implantation 6 years ago. Postoperatively the visual acuity was recorded as $6 / 9$ but bullous keratopathy developed and a corneal inlay had to be inserted 8 months ago. The eye is now quiet with a visual acuity of $6 / 24$ part.

\section{LESS SERIOUS CORNEAL CHANGES}

Less serious corneal changes occurred in nine eyes (1 3.2 per cent.) with a further case in which corneal dystrophy had been noticed before surgery:

Case II 7 years Io months since implantation. Epithelial and stromal corneal oedema some 2 to $3 \mathrm{~mm}$. from limbus in temporal cornea adjacent to temporal corneal incision overlying feet of implant. Visual acuity $6 / 12$ corrected.

Case 125 years 8 months since implantation. Limbal nasal corneal oedema overlying feet of implant. Visual acuity $6 / 9$ corrected.

Case 136 years 9 months since implantation. Fine limbal epithelial corneal oedema extending some 2 to $3 \mathrm{~mm}$. in horse-shoe area on lower two-thirds of cornea. In addition this patient has chronic open-angle glaucoma which was noted before surgery. Intraocular pressure $32 \mathrm{~mm}$. $\mathrm{Hg}$. Visual acuity $6 / 9$.

Case 148 years 3 months since implantation. Fine epithelial oedema over temporal cornea adjacent to section overlying feet of implant. Visual acuity $6 / 5$.

Case 157 years 7 months since implantation. Slight epithelial corneal oedema is encroaching some I to $2 \mathrm{~mm}$. over nasal cornea, over the feet of implant. Visual acuity $6 / 5$ corrected.

Case 167 years 8 months since implantation. Epithelial oedema over nasal cornea and temporally overlying feet of implant. In addition this patient has 80 per cent. peripheral anterior synechiae with an intraocular pressure of $27 \mathrm{~mm}$. Hg. Visual acuity 6/6.

Case 176 years I I months since implantation. Area of corneal epithelial oedema encroaching 3 to 4 $\mathrm{mm}$. on nasal cornea, overlying feet of implant. Visual acuity $6 / 9$.

Case 187 years 8 months since surgery. Corneal oedema encroaching some 3 to $4 \mathrm{~mm}$. on upper twothirds of cornea overlying feet of implant. There is free vitreous anterior to the implant, touching the corneal endothelium, which may be the cause of the corneal changes. Visual acuity 6/9. Gornea noted to have some oedema I 5 months earlier.

Case 82 years since implantation. Fine endothelial corneal dystrophy adjacent to limbus in lower two-thirds of cornea. In addition this patient has 50 per cent. peripheral anterior synechiae with vitreous fibrosis and an irritable eye. The visual acuity at one time postoperatively was $6 / 36$, but is now counting fingers.

In addition to the above cases, there is one further patient (Case 19) with early bullous keratopathy; some corneal oedema was noticed before implantation

There are also two cases of early Fuchs's corneal dystrophy in which a similar condition exists in the phakic fellow eye. 
The average time since implantation in the above eyes is 6.6 years, all except Case 8 having been in situ for over 5 years. It is extremely difficult to predict which corneas will cloud further into a generalized bullous keratopathy, affecting visual acuity and requiring keratoplasty or corneal inlay, but it seems probable that Cases I 7 and I 8 will ultimately cloud further. However, the majority of the above cases still retain a good visual acuity.

The duration of implantation seems to be the common denominator in the cases with corneal changes. The changes usually seem to be related to an area overlying the feet of the implant (Figure)

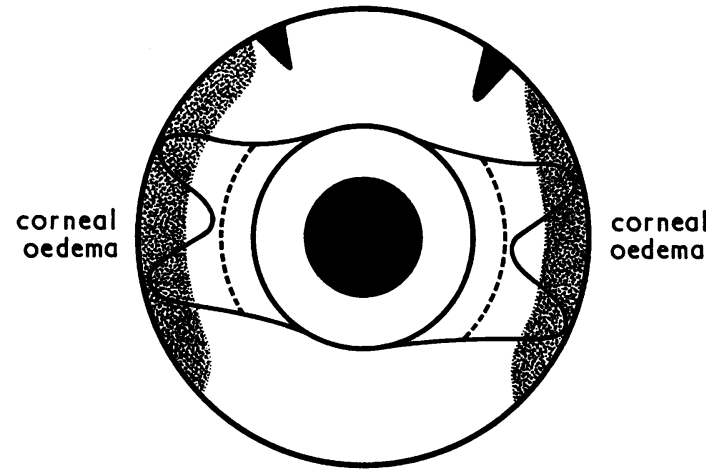

FIGURE Diagrammatic representation of typical relationship of corneal oedema to the feet of a Choyce Mark VIII implant

where the implant is lodged in the angle of the anterior chamber, although none of the above cases had corneal endothelial contact with the implant. However, four out of the eight cases (Nos I I-I 8 and No. 8) had one or two feet of the implant lodged on the ciliary body. Three cases had free vitreous in the anterior chamber touching the endothelium, and the average amount of peripheral anterior synechiae present was 46 per cent., well above the average for the whole series of 30 per cent.

The incidence of serious corneal changes is $1 \cdot 5$ per cent. and that of less serious corneal changes I 3.2 per cent. (excluding the cases of Fuchs's dystrophy and the case of preoperative corneal dystrophy).

\section{RETINAL DETAGHMENT}

This has not occurred in this series. As dilatation of the pupil could be effected without fear of dislocation of the lens, examination of the periphery of the retina could be carried out satisfactorily.

\section{ANTERIOR AND POSTERIOR UVEITIS}

Evidence of old or active uveitis was looked for on the slit-lamp microscope in view of the theoretical possibility that the relationship of the implant to the iris and ciliary body could irritate these structures giving rise to uveitis: as could gradual breakdown of the polymethyl methacrylate (Perspex C.Q.) material of the implant. A few white cells in the aqueous were noted in several cases with no other sign of an active uveitis (this is a common finding after cataract surgery without intraocular lenses).

Two eyes had suffered severe uveitis after implantation necessitating systemic and topical steroids:

Case 8 One foot of the implant is lying on the ciliary body. The angle shows 50 per cent. peripheral anterior synechiae, and extensive vitreous fibrosis. Visual acuity counting fingers.

Case 9 In this eye there is a membrane from the implant to the cornea. The eye is irritable and photophobic. Visual acuity $6 / 36$.

The incidence of severe postoperative uveitis is 3 per cent. and has evidently caused marked loss of visual function in the cases affected.

DISTORTION OF THE PUPIL

Elliptical distortion of the pupil in the meridian of insertion of the implant occurred in seventeen eyes. However, apart from affecting the pupillary reaction to light and miotics, this has little visual significance. It arose through pulling over of the iris while inserting the implant.

\section{PHOTOPHOBIA}

This is a common complaint amongst aphakics with or without intraocular prostheses, and is easily overcome by the use of tinted spectacles. In this series it occurred in ten cases but was very severe in only three.

\section{FUNDOSCOPY}

This was carried out with the direct and indirect ophthalmoscope. Senile macular degeneration was noted in four eyes and macular oedema was noted in a further four eyes-an overall incidence of macular changes giving rise to reduced visual acuity of $\mathrm{I} \cdot 7$ per cent., with an incidence of 5.9 per cent. macular oedema. Gehring (1968) noted macular oedema in some 7.6 per cent. of 236 cases of intracapsular cataract extraction without implantation, and Binkhorst and Leonard (1967) reported macular disorders which were responsible for a visual acuity of $6 / 12$ or less in fifteen out of seventy cases of secondary iris clip lens implantation, an incidence of $2 \mathrm{I}$ per cent.

\section{PATHOLOGICAL CHANGES IN THE ANTERIOR CHAMBER}

All patients, excluding only those with cloudy corneas or with corneal inlays, were examined with the Goldmann single-mirror gonioprism and the Haag- 
Streit 900 slit lamp. The position of the implant, its relation to the structures of the angle, and the presence and amount of peripheral anterior synechiae were assessed. Apart from those cases in which there was a note in the hospital records of the gonioscopic appearance of the angle after the primary cataract surgery, it is not possible to be certain in this examination which pathological changes are exclusively caused by the second-stage implantation. In some cases the peripheral anterior synechiae were undoubtedly present after the initial cataract surgery.

The average percentage of peripheral anterior synechiae present was 30 per cent., sixteen eyes having 5 per cent. peripheral anterior synechiae or less. In nineteen eyes the feet of the implant passed through a peripheral iridectomy to lie on or in the ciliary body. The majority of the other eyes had the feet of the implant firmly embedded in the last roll of the iris. There was no eye in which the corneal endothelium was in contact with the implant and all the implants appeared to be firmly in place.

Peripheral anterior synechiae were often present between the feet of the implant. Pearce (1972), reviewing Binkhorst's personal series of primary implantation of iris clip lenses in aphakia, found that in only fourteen of the 72 cases were peripheral anterior synechiae present in the angle; the other cases were free of angle changes and the average peripheral anterior synechiae was only 17 per cent., so that the anterior chamber angle-fixation type of lens seems to be more likely to cause pathological changes in the angle of the anterior chamber than the iris clip lens.

\section{INTRAOCULAR PRESSURE}

This was measured with the Goldmann applanation tonometer on the Haag-Streit 900 slit lamp.

The average intraocular pressure was $19 \mathrm{~mm} . \mathrm{Hg}$ (range 8 to 40). Six patients had an intraocular pressure of more than $22 \mathrm{~mm}$. $\mathrm{Hg}$; in two of them pathological cupping of the optic discs had been noticed before implantation.

Case 20 Old traumatic cataract. Intraocular pressure $28 \mathrm{~mm}$. $\mathrm{Hg}$ with more than 50 per cent. peripheral anterior synechiae. Optic disc normal.

Case 13 Pathological cupping of optic disc noted before implantation. Intraocular pressure $32 \mathrm{~mm}$. $\mathrm{Hg}$ with 80 per cent. peripheral anterior synechiae. Angle noted to be open after initial cataract surgery. Patient apparently suffering from chronic open-angle glaucoma which had now become compound because of the angle pathology.

Case 21 Pathological cupping of disc noted in 1955. Glaucomato-cyclitic crises required a basal iridencleisis in 1955. Intraocular pressure now $40 \mathrm{~mm}$. $\mathrm{Hg}$ with 80 per cent. peripheral anterior synechiae.
Case 22 Angle noted to be open after initial cataract surgery. Intraocular pressure now $32 \mathrm{~mm}$. $\mathrm{Hg}$ with 60 per cent. peripheral anterior synechiae. Optic disc normal.

Case I Peripheral anterior synechiae noted between peripheral iridectomies after cataract surgery. Intraocular pressure now $27 \mathrm{~mm}$. $\mathrm{Hg}$ with 80 per cent. peripheral anterior synechiae. Some vitreous lost at implantation. Optic disc normal.

Case 23 Intraocular pressure $26 \mathrm{~mm}$. $\mathrm{Hg}$ with 5 per cent. peripheral anterior synechiae and normal optic disc.

The eye of Case 23 probably has ocular hypertension, but in the eyes of Cases 13 and 21 the high intraocular pressure is very likely related to the angle changes. Apart from the one patient with 5 per cent. peripheral anterior synechiae (Case 23), there seems to be some relation between the high intraocular pressure and the degree of angle pathology. Several of the above patients had failed to keep their follow-up appointment, and attended for examination by the author only after an absence of several years. This emphasizes the importance of regular applanation tonometry and gonioscopy in patients with anterior chamber lens implants.

\section{VISUAL ACUITY}

This was tested with a Snellen's Chart, both with and without spectacle correction where necessary. Refraction was performed where no up-to-date readings were available. Details of the correcting power of the lens and the visual acuity recorded after initial cataract surgery were obtained from the hospital and private records of the patients. The average corrected visual acuity was $6 / 6$ after primary cataract surgery. Now the average uncorrected visual acuity is better than $6 / 24$, only four patients having less than $6 / 60$ vision, and the average corrected vision is $6 / 9$.

Two patients (Case 24 with a corneal inlay for bullous keratopathy, which was noted before the insertion of a Mark VIII implant, and Case 8 with corneal dystrophy and vitreous fibrosis) have no useful vision (an incidence of 3 per cent.). 58 cases ( 85 per cent.) have a corrected visual acuity of $6 / 12$ or better, and 49 cases (73 per cent.) have a visual acuity of $6 / 9$ or better.

Pearce (1972) found that, in Binkhorst's series of primary implantations of iris clip lenses, 72 per cent. of cases with implants in situ for 5 years or more had a visual acuity of $6 / 9$ or better, which emphasizes the generally good long-term visual results in both the Choyce Mark VIII anterior chamber lens and the Binkhorst iris clip lens when used to correct aphakia.

REFRACTIVE ERROR AFTER GATARACT SURGERY AND BEFORE AND AFTER IMPLANTATION OF THE CHOYCE MARK VIII IMPLANT

The refractive error was noted from the hospital 
records of the patients and when available the spherical and cylindrical component after primary cataract surgery was recorded.

The average spherical correction required was + or - I dioptre (range o to 4 ). 42 per cent. of cases required 0.5 dioptre or less to obtain their maximum visual acuity.

The average cylindrical power of the correcting lens, from the most recent refraction, is $\mathrm{I} \cdot 95$ dioptres (range 0 to $4 \cdot 5$ ).

These findings emphasize the generally satisfactory situation obtained if a standard power lenticulus of the implant is used, and an optical power equivalent to I I dioptres in a spectacle correction seems to be generally satisfactory. The average increase in astigmatism of 0.5 dioptres after implantation can be ascribed to further corneal astigmatism from the temporal corneal incision and also in some cases to tilting of the implant. There seems to be little point in having the optical portion of the lens ground accurately to the power of the aphakic refraction in most cases, as this increases the cost of the implant and also may cause delays.

\section{BINOGULAR FUNCTION}

Orthoptic assessment was made in all cases. Most patients with reasonable vision in both eyes had good voluntary convergence with slight to moderate exophoria which was marked for near fixation. Of the 42 patients who had adequate vision to allow testing in both eyes, 32 ( 76 per cent.) had some degree of stereopsis, thereby supporting the opinion of Girard and others (1962) that intraocular lenses give a better chance of binocular function than contact lenses.

In Binkhorst's series, 72 per cent. had some degree of stereopsis (Pearce, 1972).

\section{Discussion}

Long-term advantages of anterior chamber lenses in cases of aphakia

To keep this discussion in perspective it should be remembered that these patients have not suffered the problems associated with the regular use of aphakic contact lenses. A patient who has had a successful uniocular cataract extraction who is unable to tolerate contact lenses and has an uncorrected visual acuity of only counting fingers, considers the operation to have been a failure from the only point of view that he can appreciate, viz. the visual function. Ridley (1970), in his series of Tripod II anterior chamber implants, noted a visual acuity of $6 / 9$ or better in 89 per cent of cases operated on since 1960 , and in the present series the average corrected visual acuity is $6 / 9$ in patients in which the average time since implantation has been 5.5 years. During the examin- ation of patients, eleven of the earlier Mark V and Mark VII Choyce implant cases were examined; of these $8 \mathrm{I}$ per cent. had enjoyed a vision of $6 / \mathrm{I} 2$ corrected, or better, for over io years.

Corneal oedema in eyes with the Choyce Mark VIII implant over-lie the feet of the implant, as shown in the Figure (p. I02); in the case with temporal corneal oedema this occurs at the site of the second incision into the anterior chamber. Twostage implantation by Choyce's method means that some two-thirds of the cornea will have been incised, possibly increasing the chance of corneal changes. Some of these corneal changes may remain static for many more years, but others may cloud in a matter of months. Ridley (1970) now favours singlestage implantation with cataract extraction. Choyce is now carrying out a series of primary implantation cases, although he still feels that secondary implantation is preferable, providing postoperative ocular reaction is low (Choyce, I972).

The many theoretical advantages of primary implantation include:

(I) One anaesthetic only with reduced morbidity and mortality, especially in the elderly patients who require reduced hospitalization and convalescence.

(2) Reduced likelihood of postoperative divergence of the aphakic eye with problems in regaining binocular function.

(3) Smaller total corneo-scleral incision with less likelihood of damage to the endothelium and reduced corneal astigmatism.

(4) Fewer pathological changes in the angle. Some of the eyes gonioscoped had been found to have a considerable increase in peripheral anterior synechiae after implantation.

Against primary implantation is the absence of visual and refractive assessment of the aphakic eye and the possibility of trying a contact lens which is feasible for patients. However, this investigation has shown that the majority of patients achieve reasonable vision with a standard power implant so that preoperative refraction seems to be of little importance. Choyce feels that the most powerful factor against primary implantation is that there is a greater ocular reaction, i.e. a postoperative uveitis.

Binkhorst (1973), in prescribing his iris clip lens, calculates the power of the lens from oculometric data such as that provided by keratometry, spectacle correction, anterior chamber depth, and ultrasound measurements of the actual length of the eye.

Bullous keratopathy in a case with anterior chamber angle fixation probably requires removal of the prosthesis as well as a perforating keratoplasty; whereas with the iris clip lens, providing the loops of the lens are well away from the endothelium, bullous keratopathy can be treated with penetrating keratoplasty leaving the implant in situ, the pupillary lens acting as an obturator in the pupil and reducing 
vitreous complications at surgery. I have performed penetrating keratoplasty after primary implantation of an iris clip lens in several cases with minimal operative complications. Binkhorst (1973) feels that the prognosis of perforating keratoplasty in an aphakic eye could be enormously improved with a lens implant of the pupillary iris clip type.

\section{Glaucoma and pathological changes in the angle of the anterior chamber}

In this series there is a high average incidence ( 30 per cent.) of peripheral anterior synechiae and there is a probable relationship between raised intraocular pressure and pathology in the angle; this emphasizes the need for adequate peripheral iridectomies and pre- and postoperative applanation tonometry and gonioscopy. The Binkhorst type of lens seems to be associated with less postoperative pathology in the angle. Possibly primary implantation of an anterior chamber fixation lens may reduce this problem.

\section{Operative and postoperative problems of implantation}

The general incidence of these complications was small, being limited to vitreous loss and postoperative uveitis in a few cases. No eye showed endothelial contact on gonioscopy.

Binkhorst (1973) observed that endothelial contact with intraocular implants should be prevented by all means. Morgan (1970), who has made pathological studies of 27 eyes with anterior chamber implants of various types, noted secondary glaucoma after angle closure by synechiae in ten cases. It was interesting that many eyes had a foot of the haptic portion of the implant lying on or in the ciliary body with no apparent irritation of the eye.

The configuration of the lens in the Mark VIII design keeps it well away from the corneal endothelium; this supports Choyce's claim that the Mark VIII implant is an appreciable refinement of the original Strampelli type of lens (Choyce, 1970).

Of the eleven eyes with the earlier Mark V and Mark VII Choyce anterior chamber implant, two showed bullous keratopathy and another five had less extensive corneal epithelial oedema but the patients retained good visual acuity in the affected eye.

\section{Conclusions}

The last few years have seen an increased enthusiasm for the use of intraocular lenses for the correction of unilateral aphakia. Most workers in North America and Europe, however, seem to be using the pupillary lens of Binkhorst, either the four- or the two-loop design.

The Choyce Mark VIII implant is particularly valuable in the treatment of unilateral aphakia in patients unable to tolerate contact lenses, especially where there is free vitreous in the anterior chamber making the use of a pupillary lens difficult. Cases of traumatic cataract and those with large congenital, surgical, or traumatic colobomata of the iris are also very suitable for this type of implant.

Bullous keratopathy and corneal epithelial oedema occur with all types of anterior chamber and pupillary lens implant, but the incidence on the whole is low and we should not forget that these complications occur in routine cataract surgery without the implantation of a prosthesis. Similarly, postoperative and late oedematous maculopathy after cataract surgery occurs both with and without intraocular implants. Binkhorst (1973) stated that oedematous maculopathy was observed in 26 eyes ( 3 per cent.) of his series of 865 implantations, but his current thinking is that the incidence of macular oedema is higher after intracapsular than after extracapsular extraction; while the incidence may be higher after implantation, it cannot be said that macular oedema is a complication peculiar to either anterior chamber or pupillary lenses.

It would seem advisable to avoid anterior chamber fixation lenses in cases with pre-existing narrow angles, extensive preoperative peripheral anterior synechiae, and pre-existing open-angle glaucoma in view of the danger of further angle damage after implantation.

In conclusion I should like to emphasize that the general visual results, including binocular function and patient satisfaction, are excellent in the majority of cases examined.

\section{Summary}

68 eyes in which Choyce Mark VIII anterior chamber lenses had been implanted were examined, all surgery having been performed by Mr. Choyce. The average time since implantation was 5.5 years and in 85 per cent. of eyes the corrected visual acuity was $6 / 12$ or better. Bullous keratopathy, corneal epithelial oedema, and peripheral anterior synechiae giving rise to glaucoma were the most serious complications. Visual result, binocular function, and patient satisfaction were generally very good.

My thanks are due to Mr. D.P. Choyce for freely allowing me to examine his patients and his records, to his Secretary, Mrs. M. Mazirel, to Mrs. A. Colbourne for typing the paper, and to Mr. M.J. Roper-Hall for his helpful criticisms

\section{References}

BarRaQUer, J. (1970) Trans. ophthal. Soc. U.K., 90, 73I (in discussion of Dallas) 
Binkhorst, c. D. (1973) Trans. Amer. Acad. Ophthal. Otolaryng., 77, 592, 6r o, 6 14, 6r 6 and LEONARD, P. A. M. (1967) Ibid., 64, 947

bonnet, R., massin, M., and Gerhakd, J. P. (1966) "Rapport Annuel" in Bull. Soc. Ophtal. Fr. (numéro spécial), p. 173

CHOYCE, D. P. (1964) "Intraocular Lenses and Implants", p. 202. Lewis, London (1970) Proc. roy. Soc. Med., 63, 310

(1972) Personal communication

GeHring, J. R. (I968) Arch. Ophthal. (Chicago), 80, 626

GIRARD, L. J., FRIEDMAN, B., MOORE, C. D., BLAU, R. I., BINKHORST, G. D., and GobIN, M. N. (I962) Ibid., 68, 762

Kelly, T. s. B., and BUTLER, D. (I97I) Trans. ophthal. Soc. U.K., 91, 75

MILLS, P. V., and LEWIS, E. M. T. (I971) Brit. 7. Ophthal., 55, 116

MORgan, G. (1970) Proc. roy. Soc. Med., 63, 317

PEARCE, J. L. (1972) Brit. J. Ophthal., 56, 319

RIDley, H. (1970) Proc. roy. Soc. Med., 63, 309

RUBEN, M. (197I) Trans. ophthal. Soc. U.K., 91, 59

STANWORTH, A. (1972) Brit. orthopt. J., 29, 8

WALKER, W. MARTIN (1968) Quoted by DAVIES, J., and PANTER, M. (ig68) Ibid., 25, 75 